\author{
Y \\ e-Boletim de Física \\ International Centre for Physics \\ Instituto de Física, Universidade de Brasília \\ Ano VIII, Setembro $2020 \bullet$ http://periodicos.unb.br/index.php/e-bfis • eBFIS 9 9201-1(2020)
}

\title{
ESTUDO DE ESTABILIDADE DE PEQUENOS CLUSTERS MOLECULARES DE ÁGUA
}

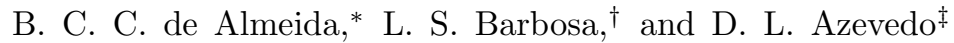 \\ Instituto de Física, Universidade de Brasília, 70910-900, Brasília, DF, Brasil
}

\begin{abstract}
A água apresenta muitas propriedades diferentes da maioria dos líquidos, por esta razão, é muito importante se estudar o comportamento da água em condições diferentes das normais de temperatura e pressão. Neste trabalho utilizamos o método inteiramente quântico da teoria do funcional da densidade(DFT) para investigar o que ocorre com a estabilidade de pequenos aglomerados de água quando colocados sob um intenso campo elétrico externo. Sob estas condições, obtivemos algumas propriedades, tais como: geometria de equilíbrio, energia de ligação, orbital ocupado de mais alta energia(HOMO), orbital desocupado de menor energia(LUMO) e frequências vibracionais. A partir do estado fundamental dos clusters na ausência de campo elétrico, aumentamos de forma gradativa o campo elétrico externo e verificamos as conformações do sistema e sua estabilidade energética. Para valores específicos da intensidade do campo elétrico(0.003 e 0.004 u.a) observamos que ocorreram transições de fase estruturais nos clusters que foram bem caracterizadas tanto dos pontos de vistas de energia quanto de orientação em relação ao campo externo.
\end{abstract}

Keywords: clusters moleculares; transição estrutural de fase; dímero; trímero; DFT

\section{INTRODUÇÃO}

A nanotecnologia é uma abordagem multidisciplinar que permite manipular sistemas atômicos e moleculares, portanto, permite desenhar novas estruturas, com novas propriedades físicas, químicas e biológicas provenientes desse novo arranjo [1].

Os pequenos clusters moleculares (2 a 4 moléculas) são aglomerados de tamanho nanométrico. As suas propriedades estão diretamente ligadas a forma que suas moléculas estão organizadas. Ao estudarmos pequenos clusters, temos a oportunidade de aprofundar a investigação a nível molecular e quando possível inferir sobre propriedades microscópicas do sistema. De fato, estes clusters servem como um protótipo para um estudo das interações entre moléculas de água, e isso pode ajudar a entender a natureza complexa de corpos extensos como a água em seu estado líquido, sólido, gasoso, entre outros. Nesse sentido, buscamos compreender alguns comportamentos anômalos da água. O dímero e o trímero são as estrutura mais simples que podem ser formadas a partir de moléculas de água, e seu processo de formação pode dar origem a alguns novos comportamentos.Portanto, iniciamos nossa investigação a partir destas estruturas. A geometria molecular do dímero e do trímero já foi estu-

\footnotetext{
* brunna.corcino@gmail.com

$\dagger$ leofis94@gmail.com

$\ddagger$ david888azv@gmail.com
}

dada em outros trabalhos, 24 4. Desta forma, o primeiro passo foi, partir dessa geometria inicial, realizar uma relaxação completa com o método da teoria do funcional da densidade. Após o que comparamos-as distâncias intermoleculares com resultados já conhecidas experimentalmente e/ou outros trabalhos publicados. Analisamos também a energia de ligação, também chamada de Binding Energy, id est a energia necessária para manter os átomos ou clusters de moléculas coes(o)as, em outras palavras, a quantidade de trabalho que deve ser realizado sobre o sistema para dissociá-lo em seus constituintes. Por fim, também analisamos a estabilidade dos clusters através de suas frequências vibracionais. Este artigo está dividido conforme segue. Na seção $I I$ apresentamos as metodologias utilizadas em nossos cálculos. Na seção III analisamos as propriedades dos dímeros e dos trímeros com suas respectivas energias antes e após a aplicação do campo elétrico externo. E por fim, na seção $I V$ apresentaremos algumas conclusões a respeito destes resultados.

\section{MÉTODOS COMPUTACIONAIS}

Neste trabalho utilizamos um método inteiramente quântico de muitos corpos baseado na teoria do funcional da densidade (DFT - Density Functional Theory) para o cálculo de várias propriedades. O programa utilizado é o módulo $\mathrm{DMol}^{3}$ [5, 6] do pacote Biovia Materials Studio. O DFT é um método ab initio e baseia-se no fato de que todas as propriedades do estado fundamental de 
um sistema eletrônico são funcionais da densidade $\rho(r)$ 7. Nesta metodologia, a expressão para a energia total do sistema é escrita como

$$
E_{t}[\rho]=T[\rho]+U[\rho]+E_{x c}[\rho]
$$

ou

$$
E[\rho]=\sum_{i=1}^{N} \epsilon_{i}+E_{x c}[\rho]-\frac{1}{2} \iint \frac{\rho(\vec{r}) \rho\left(\overrightarrow{r^{\prime}}\right)}{\left|r-r^{\prime}\right|} d \vec{r} d \overrightarrow{r^{\prime}}+\int \rho(\vec{r}) v_{x c}\left(\overrightarrow{r^{\prime}}\right) d \vec{r}
$$

onde $v_{x c}(\vec{r})$ é o potencial de troca e correlação ( potencial fictício efetivo). A equação (2) foi proposta por KohnSham [8]. O termo $E_{x c}[\rho]$ não depende das moléculas analisadas e nem do material que constitui o meio, e por não possuir expressões exatas para o mesmo, usamos aproximações. Neste artigo usamos a aproximação $G e$ neralized Gradient Approximation (GGA), uma vez que nos dímeros analisados a distribuição eletrônica não é homogênea, visto que trata-se de um cluster e estamos lidando com distâncias intermoleculares. Desta forma, nossa expressão de energia $E_{x c}[\rho]$ é reescrita como

$$
E_{X C}^{G G A}[\rho \uparrow(\vec{r}), \rho \downarrow(\vec{r})]=\int f_{x c}(\rho \uparrow(\vec{r}), \rho \downarrow(\vec{r})), \nabla \rho \uparrow(\vec{r}), \nabla \rho \downarrow(\vec{r}) d^{3} \vec{r},
$$

onde a densidade total de spin é dada por $\rho(\vec{r})=\rho \uparrow$ $(\vec{r})+\rho \downarrow(\vec{r})$. Para determinar o valor de $f_{x c}$ da equação de energia, utiliza-se funcionais, que são expressões matemáticas das quais determina-se o valor para $f_{x c}$. Assim a energia, $E_{X C}^{G G A}$ é escrita como

$$
E_{X C}^{G G A}=E_{G G A}^{x}+E_{G G A}^{c},
$$

onde $E_{G G A}^{x}$ é a energia de troca e $E_{G G A}^{c}$ é a energia de correlação. Usamos os funcionais PBE ( $a b i n i$ tio=funcional não empírico), que utiliza somente as regras gerais da mecânica quântica, e o B3LYP (híbrido = semi-empírico). Usamos esses funcionais por tais se mostrarem mais efetivos a análise de clusters moleculares 9 12. No funcional PBE a energia de troca-correlação é escrita como

$$
E_{X C}^{P B E}(\rho)=\int \rho(\vec{r}) f_{x c}^{(P B E)}\left(r_{s}(\vec{r}), s(\vec{r}), \varsigma(\vec{r})\right) d^{3} \vec{r} .
$$

No funcional B3LYP, adiciona-se um termo de troca que é calculado pelo método de Hartree-Fock. Desta forma o funcional de troca-e correlação $E_{X C}^{B 3 L Y P}$ é dado por

$$
\begin{aligned}
& E_{x c}^{B 3 L Y P}=\left(1-a_{0}\right) E_{x}^{L S D A}+a_{0} E_{x}^{H F} \\
& +a_{x} \Delta E_{x}^{B 88}+a_{c} \Delta E_{c}^{L Y P}+\left(1-a_{c}\right) E_{c}^{V W N} .
\end{aligned}
$$

Os coeficientes $a_{0}=0.20, a_{x}=0.72$ e $a_{c}=0.81$ foram sugeridos por Becke [13]. A base utilizada foi a Double Numerical Plus Polarization DNP que é comparável em tamanho ao tipo gaussiano 6-31G (d, p). Cada um desses conjuntos de bases inclui uma função de polarização do tipo $d$ em átomos pesados e uma função de polarização do tipo $p$ em átomos de hidrogênio, refinando a precisão da simulação. Uma grade de integração fina foi usada para obtenção de geometrias de alta precisão.
Essa grade de integração controla a precisão da qual os elementos da Hamiltoniana são calculados. Os limiares utilizados para convergência adequada foram a energia máxima abaixo de $1,0 \cdot 10^{-5} \mathrm{Ha}$, a força máxima abaixo de $2,0 \cdot 10^{-3} \mathrm{Ha} / \AA$ e o deslocamento atômico máximo não superior a $5,0 \cdot 10^{-3} \AA$. Além disso, empregamos $D F T$ semi-core pseudopotentials calculations (DSPP), que é um pseudopotencial que substitui os elétrons próximos ao núcleo (elétrons de caroço) por um único potencial efetivo. A energia intermolecular, ou energia de dissociação da ligação $\Delta \mathrm{E}$, foi calculada como a diferença entre as energias total do dímero ou trímero e a soma das energias isoladas de monômeros de água. Todas as simulações foram feitas com solvatação em água (constante dielétrica $\epsilon=78,54 a . u$.) à temperatura $0 K$.

\section{ESTABILIDADE DE CLUSTERS MOLECULARES DE ÁGUA}

Clusters moleculares são um intermediário entre um corpo rígido extenso e um conjunto de partículas individuais. Em certas condições, as moléculas que compõem o cluster interagem individualmente com campos externos, mas ainda mantêm a coesão propiciada pelo cluster. Além disso, pequenos clusters podem exibir propriedades exclusivas desse regime de tamanho intermediário e essas propriedades só podem ser compreendidas pela observação de interações a nível molecular.Desta forma iremos analisar os aglomerados de água com e sem a presença de campo elétrico, pois , as ligações de hidrogênio na água podem resultar em fenômenos incomuns presente em nível microscópico, sendo este o ponto de partida da nossa investigação de comportamentos anômalos em corpos extensos de água. A mudança da nanoescala para macroescala resulta em mudanças significativas nas propriedades do material sem que seja necessário alterar as moléculas e os átomos que o constituem. Essas mudanças de propriedades surgem naturalmente quando mudamos a escala na qual realizamos a análise.

\section{A. DÍMEROS}

O dímero de água é o cluster mais simples que conseguimos formar utilizando apenas duas moléculas de água, e seu processo de formação pode ser responsável por alguns comportamentos anômalos da água [14]. Por se tratar de uma estrutura simples o dímero de água foi o primeiro aglomerado molecular a ser analisado e comparado com os dados experimentais [15] e ocorre principalmente na água em forma de vapor.

O processo de investigação da estabilidade dos dímeros, iniciou-se a partir da análise da energia de ligação, comparando-as com os respectivos valores experimentais publicados. [16]. O resultado da relaxação de geometria do presente trabalho pode ser visto na Figura 1. A energia de ligação encontrada foi de $\Delta \mathrm{E}-3.55 \mathrm{Kcal} / \mathrm{mol}$ 
e a distância $O_{1}-O_{2}$ encontrada foi de $2.82(\AA)$. Ambos os valores diz respeito a simulação ao funcional hibrido $D M o l^{3}$ - B3LYP, funcional que melhor se ajustou aos dados experimentais [15, 17]. Os valores encontra-se na Tabela []

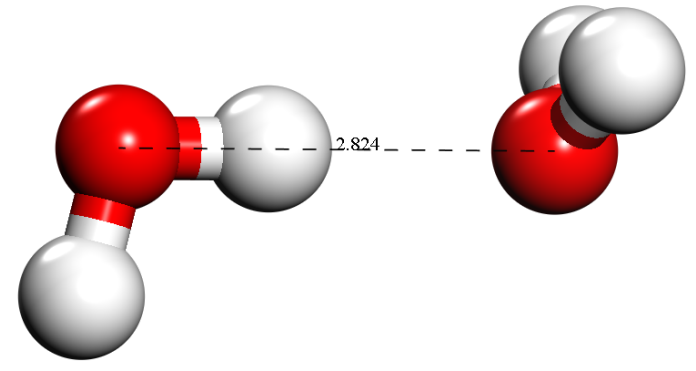

Figura 1. Dímero de água após otimização de geometria utilizando $D \mathrm{Mol}^{3}$ - B3LYP

Tabela I. Comparação entre propriedades experimentais e valores encontrados em nossas simulações para o funcional PBE e o funcional híbrido B3LYP do dímero de água sem campo elétrico. A energia de estados ligados é negativa.O valores encontrados converge para os valores esperados e atualmente aceitos 18, 19. Cabe salientar que os nossos dados refletem um trabalho teórico enquanto os da tabela representam valores experimentais.

\begin{tabular}{crrr}
\hline Propriedades Físicas & Experimental[15, [17] & PBE B3LYP \\
\hline Distancia $O_{1}-O_{2}(\AA)$ & $2.98 \pm 0.04$ & 2.81 & 2.82 \\
$\Delta \mathbf{E}(\mathrm{Kcal} / \mathbf{m o l})$ & $-3.59 \pm 0.05$ & -4.43 & -3.55 \\
\hline
\end{tabular}

O valor da energia de ligação da estrutura Trans do dímero de água sob efeito de um campo elétrico com intensidade $E=0.00 a$.u. foi $E_{b}=8.044 \mathrm{eV}$. Esse valor é menor que o da estrutura Cis do dímero de água, ou seja, essa nova configuração torna a estrutura mais estável e isso também pode ser observado a partir do gap HOMO-LUMO ( HOMO: orbital molecular ocupado de mais alta energia e LUMO: orbital molecular não ocupado de mais baixa energia). As moléculas mais difíceis de serem excitadas têm um maior gap de energia HOMO-LUMO(gapH-L), e por sua vez, as moléculas mais facéis de serem excitadas têm um gapH-L menor 20. De forma geral, quanto maior o gapH-L maior a estabilidade cinética e, quanto menor o gapH-L mais baixa a estabilidade química. As energias do HOMO e do LUMO são mostradas na Tabelaஹe o HOMO das estruturas antes e depois da transição estrutural nas Figuras 233 e 4. Nessas Figuras, para a nuvem azul representa o HOMO positivo (quadrado do módulo da parte real da função de onda) e a nuvem amarela o HOMO negativo (quadrado do módulo da parte imaginária da função de onda).
Tabela II. Orbitais de fronteira HOMO e LUMO calculados para as conformações diméricas trans e cis é o gapH-L.

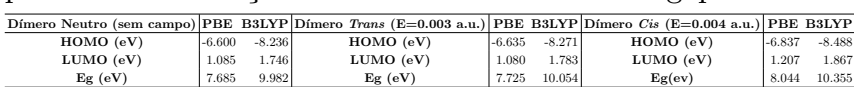

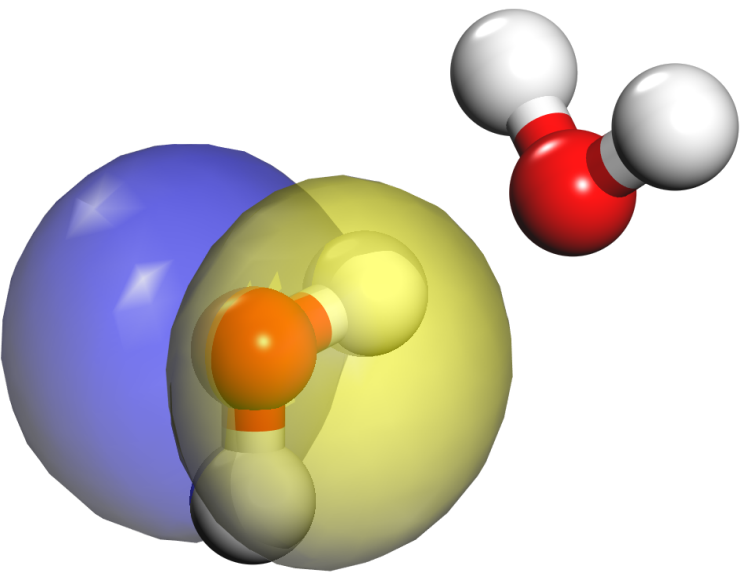

Figura 2. HOMO da estrutura Trans do dímero de água sem campo elétrico.
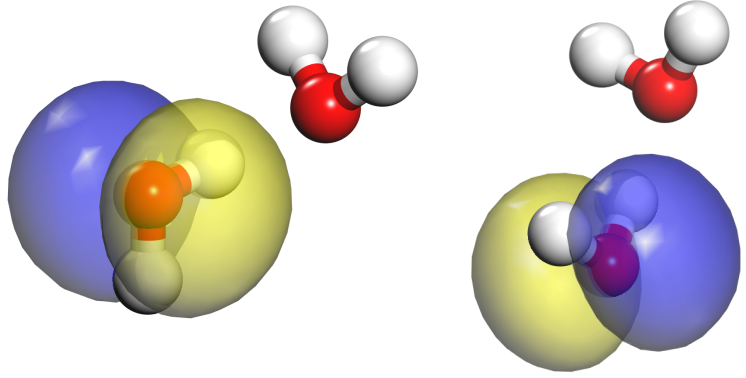

Figura 3. HOMO da estrutura Trans do dímero de água sob efeito de campo elétrico $E=$ 0.004a.u.

Figura 4. HOMO da estrutura Cis do dímero de água sob efeito de campo elétrico $E=0.004 a$.u.

Conseguimos observar que o gapH-L da estrutura Cis é maior que o da estrutura Trans. Sendo assim, concluímos que a estrutura Cis do trímero de água é a mais difícil de ser excitada, e consequentemente mais estável e menos reativa.

A estabilidade da estrutura foi testada também por meio de suas frequências vibracionais. As frequências vibracionais harmônicas são calculadas usando da matriz da derivada segunda cartesiana, também conhecida como matriz Hessiana, de um sistema molecular ou periódico 21. Geralmente usamos as matrizes Hessianas em problemas de otimização que não usam métodos Newtoni- 
anos. Aplicando para o caso de molécula (ou qualquer sistema finito periódico ou não), os elementos do Hessiano $H_{i, j}$ são dados pela derivada segunda da energia total $\Delta E$

$$
H_{i, j}=\frac{\partial^{2} \Delta E}{\partial q_{i} \partial q_{j}}
$$

de modo que $q_{i}$ é uma coordenada cartesiana generalizada de um sistema com $\mathrm{N}$ átomos, onde $1<i<3 N$. Para o caso de um sistema finito periódico, com um número muito grande de átomos, devido à periodicidade podemos fazer $H_{i, j}=H_{(i+T, j+T)}$. Assim expandindo em série de Fourier em um conjunto infinito de matrizes $3 \mathrm{~N} \times 3 \mathrm{~N}$, onde $\mathrm{N}$ agora representa o número de átomos na célula unitária, desta forma obtemos em termos de coordenadas generalizadas

$$
H_{i, j}^{\mathbf{k}}=\frac{1}{V} \sum_{\mathbf{T}} H_{i, j+\mathbf{T} e^{k, T}}
$$

$\mathbf{T}$ é o vetor de translação da rede $e^{k}$ representa o vetor na primeira zona de Brillouin. Para muitos fenômenos, o fator mais importante é a matriz Hessiana para o caso em que $k=0$, ou seja, no ponto $\Gamma$. O Hessian ponderado em massa é obtido pela divisão dos elementos Hessian pelas raízes quadradas dos produtos das massas atômicas, que é dada por

$$
F_{i, j}=\frac{H_{i, j}}{\sqrt{m_{i} m_{j}}} .
$$

Após a aplicação do campo elétrico externo aplicado ao dímero, o dipolo do cluster e/ou de seus constituintes sofrem uma rotação para se alinhar com o campo externo, esse rearranjo visa minimizar a energia do aglomerado, visto que a molécula d'água possui dipolo não-nulo. Nestas condições, as frequências obtidas a partir dos autovalores negativos são números imaginários e correspondem a formas nas quais o estado não é um mínimo da energia, e geralmente isso indica que o sistema está em um estado de transição. O modo normal correspondente a esse autovalor. Dessa forma, uma boa maneira de determinar a estabilidade das moléculas (ou um sistema periódico) é avaliar os valores das frequências, pois para valores de banda positivos temos um sistema estável e não um estado de transição. Em nossos cálculos, submetemos o dímero a um campo externo na direção- $z$, com intensidade na faixa de 0a.u. $<E_{f}<0.01 a . u$. em intervalos de 0.001a.u., e analisamos as frequências vibracionais para cada intensidade de campo. Encontramos valores positivos no intervalo de 0a.u. $<E_{f}<0.002 a . u$., porém encontramos valores negativos para o cluster enquanto colocados no campo $E=0.003 a . u$., isto é, para essa intensidade de campo detectamos um estado meta-estável no cluster. Na Figura 5 a) pode-se ver a conformação antes da transição de fase estrutural. Quando o cluster sofre a ação de campo com intensidade $E=0.004 a . u$., ocorre uma transição de fase estrutural, levando esse sistema a uma configuração mais estável energeticamente e com frequência positiva, conforme pode ser visto na Figura $5 \mathrm{~b})$.

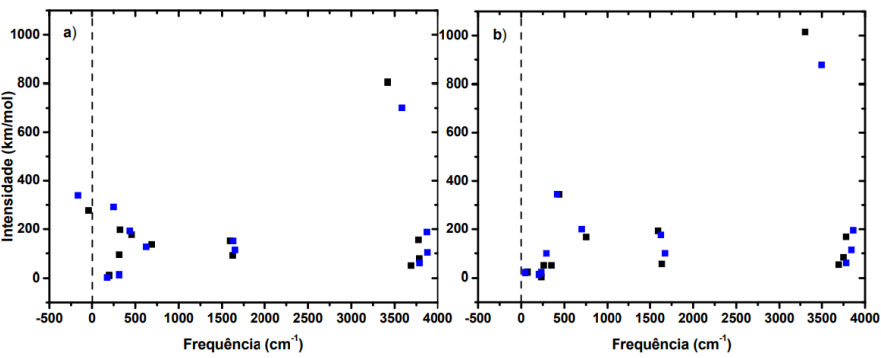

Figura 5. Frequência do dímero de água calculada com PBE (quadrado preto) e B3LYP (quadrado azul) a) Frequência do dímero de água antes da transição estrutural com $\mathrm{E}=0.003$ a.u. b) Frequência do dímero de água após a transição de fase estrutural com $\mathrm{E}=0.004$ a.u.

Todas as frequências calculadas são positivas após a transição. O resultado convergiu mesmo para o funcional híbrido B3LYP. Isso nos assegura que nosso dímero se encontra em estado estável após a transição de fase estrutural e os resultados encontrados se assemelham ao esperado na literatura [22].

E por fim, investigamos as estruturas do dímero que tiveram as frequências negativas, e para uma faixa especifica de campo 0.003a.u. $<E_{f}<0.004 a . u$. detectamos uma transição estrutural de fase do dímero que passou de uma conformação Trans para Cis, conforme pode ser visto na Figura 6 e na Figura 7.

\section{B. TRÍMEROS}

Estruturas como o trímero de água são encontradas de forma expressiva na água líquida e no gelo 3 .Tal estrutura é um ponto de partida para analise de pentâmeros e hexâmeros de água, que são as estruturas mais corriqueiras. A geometria de otimização calculada pode ser vista na Figura 8. Podemos observar que cada oxigênio atua como doador e receptor de uma ponte de oxigênio, formando assim uma "cadeia fechada". Esta forma de cadeia fechada é a mesma do $\left(\mathrm{H}_{2} \mathrm{O}\right)_{6}$ que é a forma mais encontrada em água liquida. 


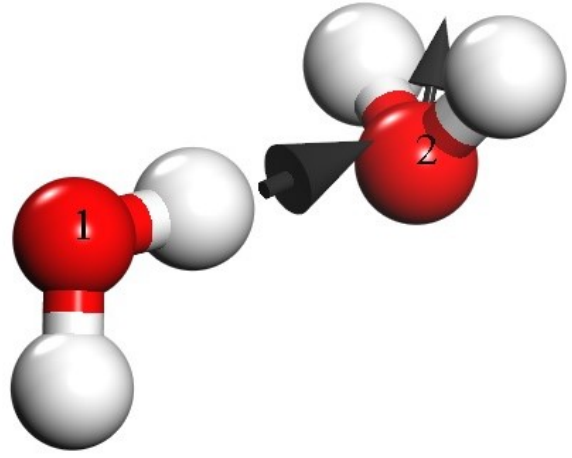

Figura 6. Estrutura Trans do dímero de água sob efeito de campo elétrico $E=0.003 a . u$.

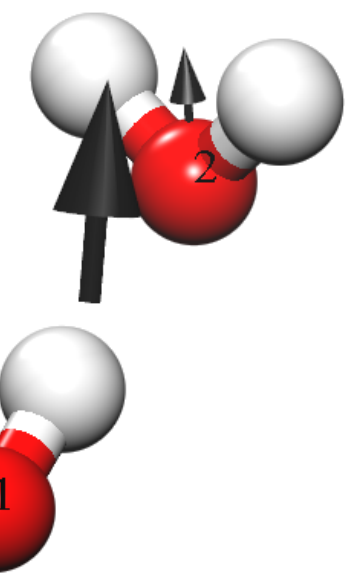

Figura 7. Estrutura Cis do dímero de água sob efeito de campo elétrico $E=0.004 a . u$.

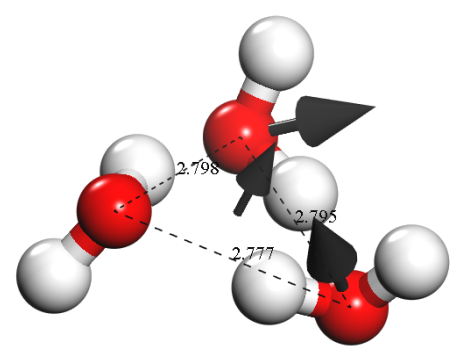

Figura 8. Trímero de água após otimização de geometria utilizando $\mathrm{DMol}^{3}$ - GGA/PBE

Ao analisamos a energia de ligação do trímero, conforme o esperado, seu valor é menor do que a do dímero, tratando-se então de uma estrutura mais coesa e por consequência, mais corriqueira na natureza. $\mathrm{O}$ valor encontrado para a energia foi de $\Delta \mathrm{E}-7.856 \mathrm{Kcal} / \mathrm{mol}$. Analisarmos também a distancia entre os átomos de
$O_{1}-O_{2}=2.826 \AA, O_{1}-O_{3}=2.795 \AA$ e $O_{2}-O_{3}=2.777 \AA$, os valores são comparados com os experimentais na Tabela III. Os valores encontrados estão em concordância com os experimentais, apontando para a confiabilidade dos nossos cálculos.

Tabela III. Comparação entre propriedades experimentais e valores encontrados em nossas simulações para o funcional PBE e o funcional híbrido B3LYP do dímero de água sem campo elétrico. A energia de estados ligados é negativa.O valores encontrados converge para os valores esperados e atualmente aceitos [3, 23, 24. Cabe salientar que os nossos dados refletem um trabalho teórico enquanto os da tabela representam valores experimentais.

\begin{tabular}{|c|c|c|c|}
\hline Propriedades Físicas & Experimental [25-27] & PBE & B3LYP \\
\hline Distancia $O_{1}-O_{2}(\AA)$ & 2.7794 & 2.798 & 2.826 \\
\hline Distancia $O_{1}-O_{3}(\AA)$ & 2.7815 & 2.795 & 2.874 \\
\hline Distancia $O_{2}-O_{3}(\AA)$ & 2.7794 & 2.777 & 2.833 \\
\hline$\Delta \mathbf{E}(\mathrm{Kcal} / \mathrm{mol})$ & -5.22 & -11.068 & -7.856 \\
\hline
\end{tabular}

Calculamos também os valores do gapH-L para os trímeros. Os valores calculados são apresentados na Tabela IV

Tabela IV. Orbitais de fronteira HOMO e LUMO calculados para a conformação do trímero trans e cis. Por exemplo, é a gapH-L em eV.

\begin{tabular}{|c|c|c|c|c|c|}
\hline râme & & Tetrâmero Trans $(\mathrm{E}=\mathbf{0}$. & \begin{tabular}{|l|} 
PBE B3LYP \\
\end{tabular} & mero $\mathrm{Cis}$ & PBE B: \\
\hline & & HOMO $(\mathrm{eV})$ & $|-6.992 \quad-8.506|$ & HOMO $(\mathrm{eV})$ & \\
\hline & -0.5 & LUMO $(e V)$ & $\left|\begin{array}{ll}0.971 & 1.591\end{array}\right|$ & LUMO $(\mathrm{eV})$ & 0.948 \\
\hline $\mathrm{Eg}(\mathrm{eV})$ & -6.037 & $\mathrm{Eg}(\mathrm{eV})$ & $\mid$\begin{tabular}{|cc}
-7.963 & -10.097
\end{tabular} & $\mathrm{Eg}(\mathrm{eV})$ & \\
\hline
\end{tabular}

Conseguimos observar que o gapH-L da estrutura Cis é maior que o da estrutura Trans. Sendo assim, concluímos que a estrutura $C i s$ do dímero de água é a mais difícil de ser excitada, e consequentemente menos reativa e sendo assim mais estável. 


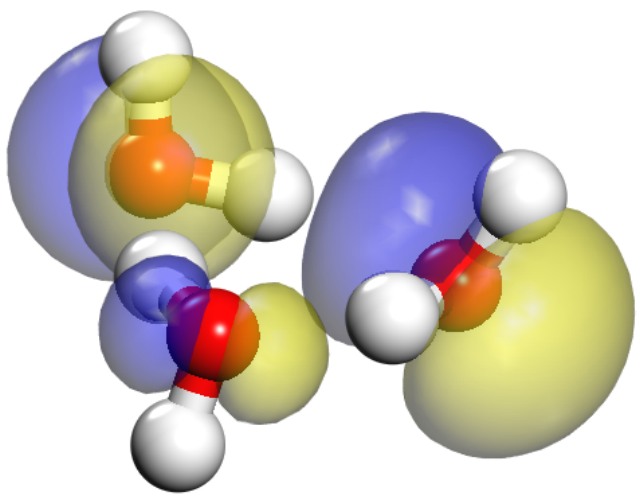

Figura 9. HOMO da estrutura Trans do trímero de água sem campo elétrico.

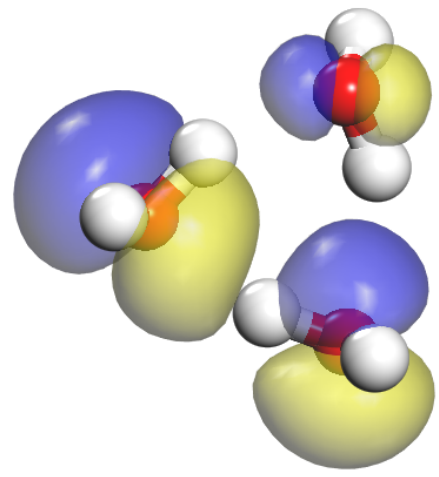

Figura 10. HOMO da estrutura Trans do trímero de água sob efeito de campo elétrico $E=0.002 a . u$
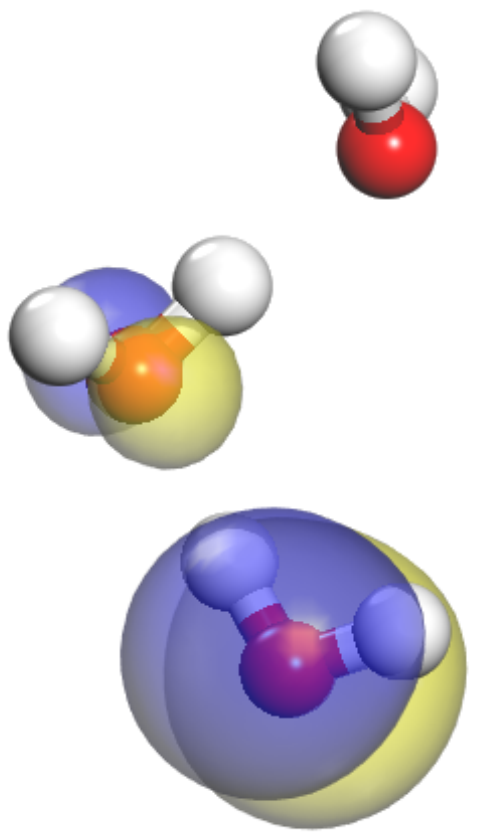

Figura 11. HOMO da estrutura $C$ is do trímero de água sob efeito de campo elétrico $E=0.003 a . u$.
Calculamos também as frequências vibracionais do trímero, tais frequências já foram estudadas por alguns autores [28 30] e desempenha um importante papel para a analise de estabilidade dos pequenos clusters. Em nossa simulação encontramos novamente uma transição estrutural para o cluster de trímero enquanto colocados no campo $E=0.002 a . u$., isto é, antes da transição da fase estrutural na conforme pode ser visto na Figura 12 a). Quando o cluster é colocado sob o campo $E=0.003 a$.u., ocorre uma transição de estrutural de fase, levando esse sistema a uma configuração estável com frequência positiva conforme pode ser visto na Figura 12 b). Em resumo, o campo elétrico novamente leva o sistema do trímero de água para uma configuração energética mais estável. Pode-se perceber que todas as frequências calculadas são positivas. Isso nos assegura que o trímero se encontra em estado estável e os resultados obtidos se assemelham aos valores esperados [3].

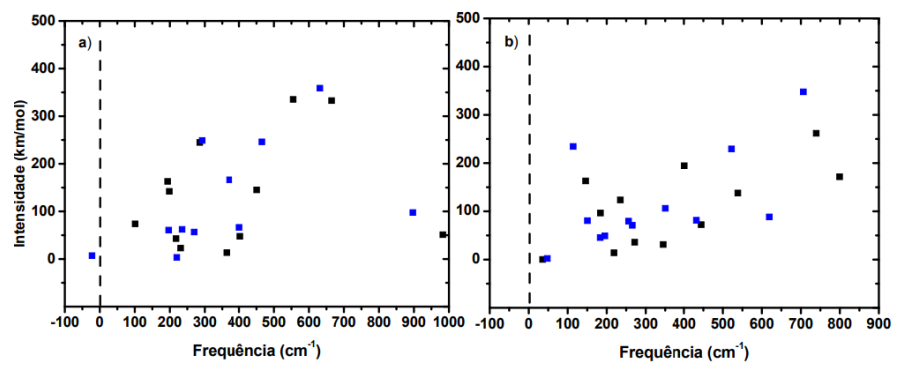

Figura 12. Frequência do trímero de água calculada com PBE (quadrado preto) e B3LYP (quadrado azul) a) Frequência do trímero de água antes da transição estrutural com $\mathrm{E}=0.002$ a.u. b) Frequência do trímero de água após a transição de fase estrutural com $\mathrm{E}=0.003$ a.u.

Iniciando novamente a investigação do comportamento anômalos da água, aplicamos ao trímero um campo elétrico e analisamos novamente sua estabilidade. E como esperado, encontramos novamente uma faixa especifica de campo 0.002a.u. $<E_{f}<0.003 a . u$. na qual detectamos uma transição de fase estrutural do trímero, que passou de uma conformação Trans para Cis, conforme pode ser visto na Figura 13 e na Figura 14. 


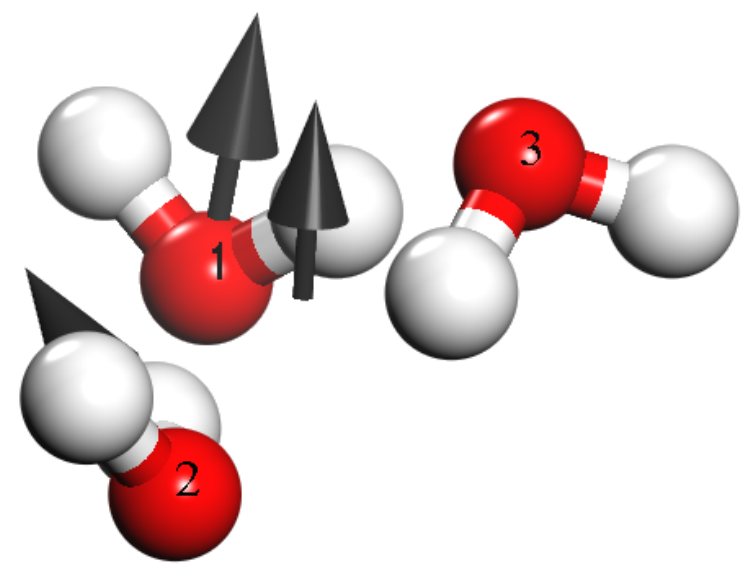

Figura 13. Estrutura Trans do trímero de água sob efeito do campo elétrico $E=0.002 a$.u.

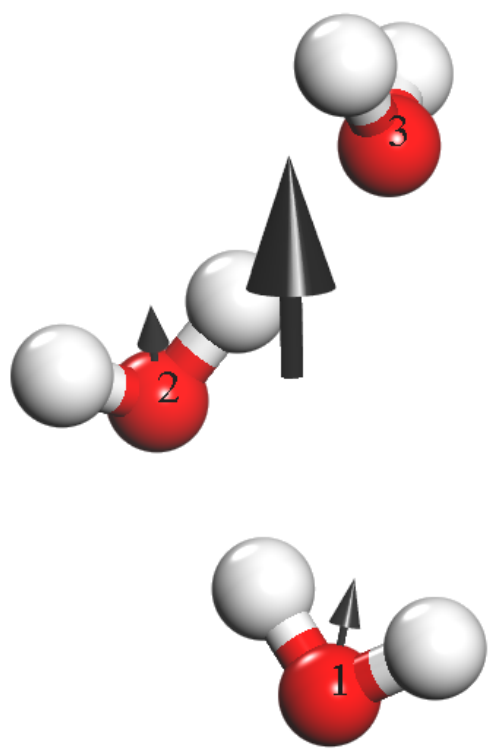

Figura 14. Estrutura $C$ is do trímero de água sob efeito do campo elétrico $E=0.003 a . u$.

\section{CONCLUSÃO}

Neste trabalho analisamos a estabilidade de dímeros e trímeros de água sob a ação de campo elétrico. Essas estruturas desempenham um papel importante na compreensão dos fenômenos microscópicos da água, para testar novos métodos computacionais, explicando características anômalas da água. Sabemos que os aglomerados moleculares não se comportam como uma única molécula, nem como um corpo rígido; portanto, devemos investigar pequenos aglomerados de uma forma mais detalhada. O estudo foi realizado através da relaxação das estruturas, de cálculos de energias de ligação e de frequências vibracionais. Os resultados obtidos através da simulação estão de acordo com alguns resultados experimentais e teóricos anteriores, corroborando assim com os resultados aqui apresentados. Após aplicarmos um campo elétrico uniforme e variarmos sua intensidade, observamos nos dois tipos de aglomerados que ocorreram transições de fase estruturais de trans para cis em ambos os dímeros e trímeros. As estruturas de aglomerados obtidas imediatamente após estas transições, são estáveis tanto do ponto de vista energética, já que possuem uma energia de ligação maior que as estruturas trans antes da transição e também são mais estáveis do ponto de vista dos orbitais de fronteira HOMO-LUMO por meio do gapH-L que é maior nas conformações pós transição de fase indicando que as estruturas cis apresentam alta estabilidade cinética e baixa reatividade química. Por meio da análise dos espectros vibracionais observamos que as estruturas cis (pós transição de fase) estão em um mínimo local com frequências positivas, ou seja, pós transição tanto o dímero quanto o trímero foram para uma configuração estável de mais baixa energia sob a influência de um campo externo. Cabe ressaltar que pelo fato de tratarmos um aglomerado e não um sistema rígido de átomos ou moléculas, os momentos dipolares de cada molécula interagem de forma individual com o campo elétrico externo e ao mesmo tempo entre si. As novas estruturas obtidas serão investigadas sob outros aspectos em trabalhos posteriores.
[1] V. V. Mitin, D. I. Sementsov, and N. Z. Vagidov. Quantum Mechanics for nanostructures. Cambridge University Press, 2010.

[2] A. Mukhopadhyay, W. T. S. Cole, and R. J. Saykally. The water dimer i: Experimental characterization. Chemical Physics Letters, 633:13-26, 2015.

[3] F. N. Keutsch, J. D. Cruzan, and R. J. Saykally. The water trimer. Chemical reviews, 103(7):2533-2578, 2003.

[4] J. R. Bordin, A. Diehl, and M. C. Barbosa. Relation between flow enhancement factor and structure for coresoftened fluids inside nanotubes. The Journal of Physical Chemistry B, 117(23):7047-7056, 2013.

[5] B. Delley. From molecules to solids with the dmol 3 approach. The Journal of chemical physics, 113(18):7756-
7764,2000

[6] B. Delley. An all-electron numerical method for solving the local density functional for polyatomic molecules. The Journal of chemical physics, 92(1):508-517, 1990.

[7] W. Hohenberg P., Kohn. Inhomogeneous electron gas. Physical Review B, 136(3):864-871, 1964.

[8] L. J. Sham W. Kohn. Self-consistent equations including exchange and correlation effects. Physical Review, 140:A1133-A1138, 1965.

[9] A. D. Becke. Becke's three parameter hybrid method using the lyp correlation functional. J. Chem. Phys, 98:5648-5652, 1993.

[10] C. Lee, W. Yang, and R. G. Parr. Development of the colle-salvetti correlation-energy formula into a functio- 
nal of the electron density. Physical review B, 37(2):785, 1988.

[11] S. H. Vosko, L. Wilk, and M. Nusair. Accurate spindependent electron liquid correlation energies for local spin density calculations: a critical analysis. Canadian Journal of physics, 58(8):1200-1211, 1980.

[12] P.J. Stephens, F.J. Devlin, C.F.N. Chabalowski, and Michael J. Frisch. Ab initio calculation of vibrational absorption and circular dichroism spectra using density functional force fields. The Journal of Physical Chemistry, 98(45):11623-11627, 1994.

[13] A. D. Becke. A multicenternumerical integration scheme for polyatomic molecules. The Journal of Chemical Physics, 88(4):2547-2553, 1988.

[14] R. Feistel, Jeremy W. Lovell-Smith, and O. Hellmuth. Virial approximation of the teos-10 equation for the fugacity of water in humid air. International Journal of Thermophysics, 36(1):44-68, 2015.

[15] A. Mukhopadhyay, W. T. S. Cole, and R. J. Saykally. The water dimer i: Experimental characterization. Chemical Physics Letters, 633:13-26, 2015.

[16] Martin W Feyereisen, David Feller, and David A Dixon. Hydrogen bond energy of the water dimer. The Journal of Physical Chemistry, 100(8):2993-2997, 1996.

[17] Charles Braun and Henning Leidecker. Rotation and vibration spectra for the h2o dimer: Theory and comparison with experimental data. The Journal of Chemical Physics, 61(8):3104-3113, 1974.

[18] Marshall D Newton and Neil R Kestner. The water dimer: Theory versus experiment. Chemical Physics Letters, 94(2):198-201, 1983.

[19] Peter A Kollman and Leland C Allen. Theory of the hydrogen bond: electronic structure and properties of the water dimer. The Journal of Chemical Physics, 51(8):3286-3293, 1969.

[20] R. G. Pearson. Absolute electronegativity and hardness: application to inorganic chemistry. Inorganic chemistry, 27(4):734-740, 1988.
[21] Paul C Cross, JC Decius, and Edgar Bright Wilson. Molecular Vibrations: The Theory of Infrared and Raman Vibrational Spectra. Cross. McGraw-Hill, 1955.

[22] J.A. Odutola and T.R. Dyke. Partially deuterated water dimers: Microwave spectra and structure. The Journal of Chemical Physics, 72(9):5062-5070, 1980.

[23] M. Mandziuk. On the tunneling splitting in a cyclic water trimer. Chemical Physics Letters, 661:263-268, 2016.

[24] B Tremblay, B Madebène, ME Alikhani, and JP Perchard. The vibrational spectrum of the water trimer: Comparison between anharmonic ab initio calculations and neon matrix infrared data between 11,000 and 90 cm- 1. Chemical Physics, 378(1-3):27-36, 2010.

[25] A. Shank, Y. Wang, A. Kaledin, B. J. Braams, and J. M. Bowman. Accurate ab initio and hybrid potential energy surfaces, intramolecular vibrational energies, and classical ir spectrum of the water dimer. The Journal of chemical physics, 130(14):144314, 2009.

[26] B. E Rocher-Casterline, L. C. Ch'ng, A. K. Mollner, and H. Reisler. Communication: Determination of the bond dissociation energy (d 0) of the water dimer,(h2o) 2, by velocity map imaging, 2011.

[27] J. E. Fowler and H. F. III Schaefer. Detailed study of the water trimer potential energy surface. Journal of the American Chemical Society, 117(1):446-452, 1995.

[28] EHT Olthof, A Van der Avoird, PES Wormer, Kun Liu, and RJ Saykally. Tunneling dynamics, symmetry, and far-infrared spectrum of the rotating water trimer. ii. calculations and experiments. The Journal of chemical physics, 105(18):8051-8063, 1996.

[29] Ad van der Avoird, EHT Olthof, and PES Wormer. Tunneling dynamics, symmetry, and far-infrared spectrum of the rotating water trimer. i. hamiltonian and qualitative model. The Journal of chemical physics, 105(18):80348050, 1996.

[30] EHT Olthof, A Van der Avoird, PES Wormer, Kun Liu, and RJ Saykally. Tunneling dynamics, symmetry, and far-infrared spectrum of the rotating water trimer. ii. calculations and experiments. The Journal of chemical physics, 105(18):8051-8063, 1996. 